\title{
Stokes matrices for the quantum differential equations of some Fano varieties
}

\author{
John Alexander Cruz Morales • \\ Marius van der Put
}

Received: 15 May 2014 / Revised: 16 June 2014 / Accepted: 18 June 2014 /

Published online: 2 December 2014

(C) Springer International Publishing AG 2014

\begin{abstract}
The classical Stokes matrices for the quantum differential equation of $\mathbb{P}^{n}$ are computed using multisummation and the 'monodromy identity'. Thus, we recover the results of D. Guzzetti that confirm Dubrovin's conjecture for projective spaces. The same method yields explicit formulas for the Stokes matrices of the quantum differential equations of smooth Fano hypersurfaces in $\mathbb{P}^{n}$ and for weighted projective spaces.
\end{abstract}

Keywords Stokes matrices · Quantum cohomology · Monodromy identity · Quantum differential equations

Mathematics Subject Classification $\quad 34 \mathrm{M} 40 \cdot 53 \mathrm{D} 45$

\section{Introduction}

For a Fano variety $X$ one can define a Frobenius structure for its cohomology and the latter induces a linear differential equation(or connection in one or more variables)

Originally since May 2013 the paper had been processed by the Central European Journal of Mathematics but it was withdrawn in April 2014 due to imposition of publishing fees and resubmitted to the European Journal of Mathematics.

JACM was supported by a Japanese Government (Monbukagakusho:MEXT) Scholarship.

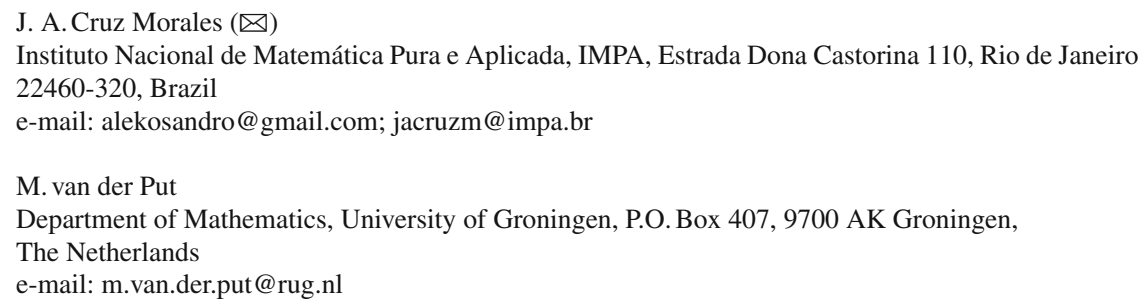


which is called the quantum differential equation of $X$. This equation reflects geometric properties of $X$ and for many varieties $X$ the quantum differential equation is explicitly known, see [5,7]. For the cases that we consider, the quantum differential equation is an ordinary linear differential equation in a complex variable $z$ and it has two singular points $z=0$ and $z=\infty$. The point $z=0$ is regular singular and the point $z=\infty$ is irregular singular. At $z=\infty$ the difference between formal (symbolic) solutions and actual solutions in sectors is measured by Stokes data. The contribution of this paper to the theory of quantum differential equations is an explicit computation of the Stokes data by means of the formalism of multisummation. This formalism is the work of many experts, see [10, Section 7.1], and in Sect. 2 we will explain how it can be used to compute the Stokes data in a purely algebraic way. We note that for a general irregular singularity there are only analytic methods for the determination of the Stokes data. Thus quantum differential equations are rather special.

In the remaining part of this introduction we sketch, for the convenience of the reader (without proof and any originality, compare $[5,8,13,14]$ ), some facts from the theory of quantum cohomology. The relation with the above Stokes data and our results concerning these are presented.

Let $X$ be a (smooth) complex projective Fano variety. Put $H^{*}(X, \mathbb{C})=$ $\bigoplus_{d \geq 0} H^{2 d}(X, \mathbb{C})$. Let $b_{1}, \ldots, b_{r}$ be a basis of $H^{2}(X, \mathbb{C})$. For $t=\sum t_{i} b_{i}$, one defines a deformation $\circ_{t}$ of the usual cup product $\circ$ on $H^{*}(X, \mathbb{C})$. This deformation is called the small quantum product. One writes formally $q_{i}=e^{t_{i}}$ and $\partial_{i}=q_{i} \partial / \partial q_{i}$. Further, $\hbar$ will denote a complex parameter. One defines a connection $\nabla$, called the DubrovinGivental connection, on the trivial vector bundle $H^{2}(X, \mathbb{C}) \times H^{*}(X, \mathbb{C}) \rightarrow H^{2}(X, \mathbb{C})$ by the formula

$$
\nabla_{\partial_{i}}=\partial_{i}-\frac{1}{\hbar} b_{i} \circ_{t}, \quad i=1, \ldots, r
$$

The quantum differential equations are the equations $\hbar \partial_{i} \Psi=b_{i} \circ_{t} \Psi$ for $i=1, \ldots, r$ and for functions $\Psi: H^{2}(X, \mathbb{C}) \rightarrow H^{*}(X, \mathbb{C})$.

Above, we have supposed $t \in H^{2}(X, \mathbb{C})$. However, it is important to consider also $t \in H^{*}(X, \mathbb{C})$. In this case the deformation of the cup product is called the big quantum product. For the corresponding 'big quantum cohomology' and 'connection' we refer to $[2,4]$.

In the sequel we restrict ourselves to the small quantum product and to the case $r=1$, i.e., the case where the quantum differential equation is an ordinary linear differential equation. For a detailed discussion we refer the reader to [5] and references therein.

A 'good Fano variety' $X$ is a Fano variety such that $D^{b} \operatorname{coh}(X)$, the derived category of the coherent sheaves on $X$, is generated as triangulated category by an exceptional collection $\left(\mathcal{E}_{i}\right)_{i=1}^{N}$. An object $\mathcal{E}$ is exceptional if $\operatorname{Ext}^{i}(\mathcal{E}, \mathcal{E})$ equals $\mathbb{C}$ for $i=0$ and equals 0 for $i>0$. Further, $\left(\mathcal{E}_{i}\right)_{i=1}^{N}$ is an exceptional collection if each $\mathcal{E}_{i}$ is exceptional and $\operatorname{Ext}^{k}\left(\mathcal{E}_{i}, \mathcal{E}_{j}\right)=0$ for any $i>j$ and any $k$. In this situation the Gram matrix $G$ of $X$ is defined by $G_{i j}=\sum_{k}(-1)^{k} \operatorname{dim} \operatorname{Ext}^{k}\left(\mathcal{E}_{i}, \mathcal{E}_{j}\right)$.

One of conjectures of Dubrovin [3] states that the Gram matrix of $X$ coincides with the Stokes matrix of the quantum differential equation of $X$ (up to a certain equivalence 
which we will make more explicit). For the complex projective space $\mathbb{P}^{n-1}$, the ordered set of line bundles $O, O(1), \ldots, O(n-1)$ is an exceptional collection and the Gram matrix $G=\left(G_{i j}\right)$ is given by $G_{i j}=\left(\begin{array}{c}n-1+j-i \\ j-i\end{array}\right)$ for $i \leq j$ and $G_{i j}=0$ otherwise. The inverse $\left(a_{i j}\right)$ of $G$, which is equivalent to $G$, has the data $a_{i j}=(-1)^{j-i}\left(\begin{array}{c}n \\ j-i\end{array}\right)$ for $i \leq j$ and $a_{i j}=0$ otherwise.

Now we will explain the relation between 'our' Stokes data and the Stokes matrix considered in quantum cohomology by Dubrovin et al. The latter we will call 'quantum Stokes matrices' and denote by $\mathrm{St}_{\mathrm{qc}}$. The irregular singularity of the quantum differential equation at $z=\infty$ has Poincaré rank 1. This implies that a given formal (or symbolic) fundamental matrix can be lifted to an actual analytic fundamental matrix on a sector at $z=\infty$ of opening slightly larger than $\pi$. Moreover, these liftings are unique. Let $\Phi_{\text {right }}$ and $\Phi_{\text {left }}$ denote two of these lifts, then $\mathrm{St}_{\mathrm{qc}}$ is defined by $\Phi_{\text {right }}=\Phi_{\text {left }} \mathrm{St}_{\mathrm{qc}}$.

The multisummation theory produces for every singular direction $d$ of the differential equation a Stokes matrix, denoted by $\mathrm{St}_{d}$. This expresses the relation between multisummation of the formal fundamental matrix left and right of the singular direction $d$. One concludes that $\mathrm{St}_{\mathrm{qc}}$ equals the ordered product $\prod_{d} \mathrm{St}_{d}$ taken over the singular directions $d$ in an interval of length $\pi$ (in fact $d \in[0,1 / 2)$ in our notation). It turns out that each $\mathrm{St}_{d}$ has only one interesting entry. The collection of these entries will be called the Stokes data. We note that 'our' Stokes data are closely related to what are called 'Stokes factors' in [8].

For the complex projective space $\mathbb{P}^{n-1}$ the conjecture of Dubrovin has been proved by Guzzetti [8]. The matrix $\mathrm{St}_{\mathrm{qc}}$ (the product $\prod_{d} \mathrm{St}_{d}$ ) is a unipotent matrix and is, a priori, rather complicated with respect to the given basis, see [8, Section 6]. This basis is changed (this is the equivalence mentioned before) by a permutation, by putting signs and the action of a braid group. The quantum differential equation lives in a family (in fact induced by the big quantum product), parameterized by $\mathbb{C}^{n} \backslash$ the diagonals, of similar equations where the singular directions at $z=\infty$ vary. The braid group action is derived from loops in this family. Guzzetti showed that $\mathrm{St}_{\mathrm{qc}}$ has, w.r.t. a new basis and up to signs, the form $\left(a_{i j}\right)$ which proves the Dubrovin's conjecture for $\mathbb{P}^{n-1}$.

In this paper we prove for the Stokes data $\left\{x_{\ell k}\right\}_{0 \leq k, \ell<n ; k \neq \ell}$ of $\mathbb{P}^{n-1}$, see Theorem 3.1, that:

- For odd $n$ and $0 \leq \ell<k$ one has $x_{\ell k}=-(-1)^{k-\ell}\left(\begin{array}{c}n \\ k-\ell\end{array}\right)$ and $x_{\ell k}=-x_{k \ell}$.

- For even $n$ and $0 \leq \ell<k$ one has $x_{\ell k}=-(-1)^{k-\ell}\left(\begin{array}{c}n \\ k-\ell\end{array}\right)$ if $k-\ell \leq n / 2$ and $x_{\ell k}=(-1)^{k-\ell}\left(\begin{array}{c}n \\ k-\ell\end{array}\right)$ if $k-\ell>n / 2$.

- For even $n$ and $0 \leq k<\ell$ one has $x_{\ell k}=(-1)^{\ell-k}\left(\begin{array}{c}n \\ \ell-k\end{array}\right)$.

Theorem 3.1 proves again Dubrovin's conjecture for $\mathbb{P}^{n-1}$ and we observe that the above matrix $\left(a_{i j}\right)$, equivalent to $\mathrm{St}_{\mathrm{qc}}$, can rather simply be expressed into the Stokes data $\left\{x_{\ell k}\right\}$. The Stokes data can be read off from the monodromy identity which compares the topological monodromy at $z=0$ with the Stokes matrices $\mathrm{St}_{d}$ and the formal monodromy at $z=\infty$. The same method leads to further results: computations of the Stokes data for weighted projective spaces (Remark 3.2 and Proposition 3.3) and for Fano hypersurfaces (Theorem 4.1). 
Recent papers on computation of quantum Stokes matrices are $[11,13,14]$. The first one proposes another proof of Dubrovin's conjecture for $\mathbb{P}^{n}$. In the other two papers quantum Stokes matrices are computed for Grassmannians (based on the results for $\left.\mathbb{P}^{n}\right)$ and for cubic surfaces.

After completing the calculations of this paper we became aware that a related discussion (from a physical point of view) to our work is presented in [15], for the case of projective spaces. However, the argument in loc.cit. concerns the computation of the Stokes matrices for the so-called $\mathrm{tt}^{*}$ equations, see [1]. The question whether these equations are related to the equations for the quantum cohomology and, in particular, whether their Stokes matrices coincide, is discussed in [6].

The paper is organized as follows. In Sect. 2 we give a brief presentation of the theory of Stokes matrices emphasizing the relevant facts for our computation. In Sect. 3 we present the explicit computation for the case of (weighted) projective spaces and in Sect. 4 we extend that computation to the case of smooth Fano hypersurfaces. In the sequel $q$ will be replaced by $z$ and the parameter $\hbar$ is taken to be 1 . We will often write $\delta$ for $z d / d z$. The quantum differential equation in operator form for $\mathbb{P}^{n-1}$ then obtains the simple form $\delta^{n}-z$.

\section{Stokes matrices and monodromy identity}

A linear differential operator of order $n$, analytic in the neighbourhood of $z=\infty$, has a scalar form

$$
\left(z \frac{d}{d z}\right)^{n}+a_{n-1}\left(z \frac{d}{d z}\right)^{n-1}+\cdots+a_{1} z \frac{d}{d z}+a_{0}
$$

with all $a_{j}$ in the field $\mathbb{C}\left(\left\{z^{-1}\right\}\right)$ of the convergent Laurent series in $z^{-1}$. The scalar operator can be transformed into a matrix differential operator $z d / d z+A$ where the entries of the matrix $A$ are in $\mathbb{C}\left(\left\{z^{-1}\right\}\right)$.

As a differential module over $\mathbb{C}\left(\left\{z^{-1}\right\}\right)$, the scalar equation above translates into a vector space $M$ of dimension $n$ over this field, equipped with a $\mathbb{C}$-linear operator $\delta_{M}$ satisfying

$$
\delta_{M}(f m)=z \frac{d}{d z}(f) \cdot m+f \delta_{M}(m)
$$

for $f \in \mathbb{C}\left(\left\{z^{-1}\right\}\right), m \in M$. Note that for a suitable basis of $M$, the matrix $A$ above is the matrix of $\delta_{M}$ with respect to this basis.

The formal classification of $M$ is the classification of the differential module $\mathbb{C}\left(\left(z^{-1}\right)\right) \otimes M$ over the field $\mathbb{C}\left(\left(z^{-1}\right)\right)$ of the formal Laurent series in $z^{-1}$. In general, a root $z^{1 / m}$ of $z$ for certain $m \geq 1$ is needed for the formulation of the classification that we describe now.

There are distinct elements $q_{1}, \ldots, q_{s} \in z^{1 / m} \mathbb{C}\left[z^{1 / m}\right]$, called the generalized eigenvalues of $M$, such that $\mathbb{C}\left(\left(z^{-1 / m}\right)\right) \otimes M$ is a direct sum of (differential) submodules $N_{1}, \ldots, N_{r}$ over $\mathbb{C}\left(\left(z^{-1 / m}\right)\right)$. The differential module $N_{j}$ has a basis such that the 
operator $\delta_{N_{j}}$ has the form $q_{j} \cdot \mathrm{id}+\ell_{j}$, where $\ell_{j}$ has entries in $\mathbb{C}$. The elements $q_{j}$ and the decomposition $\mathbb{C}\left(\left(z^{-1 / m}\right)\right) \otimes M=N_{1} \oplus \cdots \oplus N_{r}$ are unique. The elements $\ell_{j}$ are not unique.

One defines symbols $z^{\lambda}$ for every $\lambda \in \mathbb{C}, \log z$ and $e(q)$ for every $q \in$ $\bigcup_{n \geq 1} z^{1 / n} \mathbb{C}\left[z^{1 / n}\right]$, by the rules $z^{\lambda_{1}+\lambda_{2}}=z^{\lambda_{1}} z^{\lambda_{2}}, z^{0}=1, z^{1}=z, e\left(q_{1}+q_{2}\right)=$ $e\left(q_{1}\right) e\left(q_{2}\right), e(0)=1$ and $\delta\left(z^{\lambda}\right)=\lambda z^{\lambda}, \delta(\log z)=1, \delta(e(q))=q \cdot e(q)$. On a sector at $z=\infty$ these symbols have an obvious interpretation (e.g., the interpretation of $e(q)$ is $\left.e^{\int q d z / z}\right)$, but not on a full neighbourhood of $z=\infty$.

Let $\gamma$ denote the automorphism of $\bigcup_{n \geq 1} \mathbb{C}\left(\left(z^{-1 / n}\right)\right)$ defined by $\gamma z^{\lambda}=e^{2 \pi i \lambda} z^{\lambda}$ for all $\lambda \in \mathbb{Q}$. The natural action of $\gamma$ on the symbols is given by the formulas $\gamma z^{\lambda}=e^{2 \pi i \lambda} z^{\lambda}$ for all $\lambda \in \mathbb{C}, \gamma \log z=2 \pi i+\log z, \gamma e(q)=e(\gamma q)$.

Symbolic solution space. Let $U$ be the $\mathbb{C}\left(\left(z^{-1}\right)\right)$-algebra generated by these symbols. Then $U$ is a universal Picard-Vessiot ring for the differential field $\mathbb{C}\left(\left(z^{-1}\right)\right)$, which means that for every differential module $M$ over $\mathbb{C}\left(\left(z^{-1}\right)\right)$, the $\mathbb{C}$-vector space $V=\operatorname{ker}(\delta, U \otimes M)$ has the property that the obvious map $U \otimes \mathbb{C} V \mapsto U \otimes M$ is an isomorphism. Moreover, $U$ is minimal with this property and $U$ has only trivial differential ideals. The space $V$ is called the symbolic solution space of $M$. Let $b_{1}, \ldots, b_{d}$ be a basis of $M$ over $\mathbb{C}\left(\left(z^{-1}\right)\right)$. The elements of $V$ are sums $\sum_{j=1}^{d} \alpha_{j} b_{j}$, where $\alpha_{j} \in U$ are (by definition) expressions using formal power series, and the symbols $z^{\lambda}, \log z, e(q)$.

The decomposition $U=\bigoplus_{q} U_{q}$ with $U_{q}=e(q) \mathbb{C}\left(\left(z^{-1}\right)\right)\left[\left\{z^{\lambda}\right\}, \log z\right]$ induces a decomposition $V=\bigoplus_{q} V_{q}$ with $V_{q}=\operatorname{ker}\left(\delta, U_{q} \otimes M\right)$. Further, $\gamma$ acts as a $\mathbb{C}$-linear automorphism on $V$ and has the property $\gamma\left(V_{q}\right)=V_{\gamma q}$. The action of $\gamma$ on $V$ is called the formal monodromy.

Thus we have associated to $M$ a tuple $\left(V,\left\{V_{q}\right\}, \gamma\right)$ of a finite dimensional $\mathbb{C}$ vector space $V$, a subspace $V_{q}$ for every $q$ in the set of generalized eigenvalues $\bigcup_{n \geq 1} z^{1 / n} \mathbb{C}\left[z^{1 / n}\right]$, an element $\gamma \in \mathrm{GL}(V)$ such that $V=\bigoplus V_{q}$ and $\gamma V_{q}=V_{\gamma q}$ for every $q$. This construction yields in fact an equivalence of Tannakian categories, see [10] for more details.

Singular directions and multisummation. For a pair of distinct eigenvalues $(q, \widetilde{q})$, one considers the operator

$$
z \frac{d}{d z}-(q-\widetilde{q})=z \frac{d}{d z}-\left(c z^{\lambda}+\cdots\right)
$$

with $\lambda>0, c \neq 0$ and the dots are terms $* z^{\mu}$ with $0<\mu<\lambda$. The solution of the equation is $y=e^{c z^{\lambda} / \lambda+\cdots}$. Let $d \in \mathbb{R}$ stand for the direction $e^{2 \pi i d}$ at $z=\infty$. Then a real number $d$ is called a singular direction for the pair $(q, \widetilde{q})$ if and only if $c e^{2 \pi i \lambda d} / \lambda$ is real and negative.

Let $M$ be a differential module over $\mathbb{C}\left(\left\{z^{-1}\right\}\right)$. Multisummation in a direction $d$ is a $\mathbb{C}$-linear bijection $m_{d}$ from the symbolic solution space $V$ of $M$ to the space of the actual solutions of $M$ in a sector around the direction $d$. The map $m_{d}$ exists (and is unique) if $d$ is not a singular direction for any pair $(q, \widetilde{q})$ of eigenvalues of $M$. 
Stokes maps. Let a differential module $M$ over $\mathbb{C}\left(\left\{z^{-1}\right\}\right)$ be given and let $\left(V,\left\{V_{q}\right\}, \gamma\right)$ be the tuple corresponding to $\mathbb{C}\left(\left(z^{-1}\right)\right) \otimes M$. Let $d$ be a direction. Then the Stokes map $\mathrm{St}_{d}$ for this direction has the form $\mathrm{St}_{d}=1+\sum M_{d, q, \tilde{q}}$, where the sum is taken over all pairs $(q, \widetilde{q})$ such that $V_{q}, V_{\widetilde{q}} \neq 0$ (i.e., $q$ and $\widetilde{q}$ are eigenvalues for $\left.M\right), d$ is a singular direction for $(q, \widetilde{q})$ and

$$
M_{d, q, \tilde{q}}: V \stackrel{\text { projection }}{\longrightarrow} V_{q} \stackrel{\text { linear }}{\longrightarrow} V_{\widetilde{q}} \stackrel{\text { inclusion }}{\longrightarrow} V .
$$

This Stokes map is obtained by comparing the multisummation maps $m_{d-\epsilon}$ and $m_{d+\epsilon}$ (with small enough $\epsilon>0$ ) from $V$ to actual solutions of the differential equation in a sector around the direction $d$. Further, $\gamma^{-1} \mathrm{St}_{d} \gamma=\mathrm{St}_{d+1}$. We note that a direction $d$ can be singular for more than one pair $(q, \widetilde{q})$.

For a given differential module $M$ over $\mathbb{C}\left(\left\{z^{-1}\right\}\right)$, there is an algorithm computing the tuple $\left(V,\left\{V_{q}\right\}, \gamma\right)$. The entries of the Stokes maps can be expressed as certain involved integrals and, in general, these cannot be made explicit.

Now we have associated to a differential module $M$ over $\mathbb{C}\left(\left\{z^{-1}\right\}\right)$ a tuple $\left(V,\left\{V_{q}\right\}, \gamma,\left\{\mathrm{St}_{d}\right\}\right)$ with the properties stated above. This yields an equivalence between the Tannakian categories of the differential modules over $\mathbb{C}\left(\left\{z^{-1}\right\}\right)$ and the category of these tuples, see [10, Theorem 9.11].

Change of variables. The inclusion $K=\mathbb{C}\left(\left\{z^{-1}\right\}\right) \mapsto K_{n}=\mathbb{C}\left(\left\{u^{-1}\right\}\right)$ with $z=u^{n}$ and $n>1$ induces a functor which associates to a differential module $M$ over $K$ the differential module $K_{n} \otimes M$ over $K_{n}$. The corresponding morphism between tuples maps a tuple $\left(V,\left\{V_{q}\right\}, \gamma,\left\{\mathrm{St}_{d}\right\}\right)$ to the tuple $\left(V,\left\{V_{\widetilde{q}}\right\}, \widetilde{\gamma},\left\{\widetilde{\mathrm{St}}_{d}\right\}\right)$. It can be verified that $V_{\widetilde{q}}=V_{q}$ for $\widetilde{q}(u)=q\left(u^{n}\right), \widetilde{\gamma}=\gamma^{n}$ and $\widetilde{\mathrm{St}}_{d}=\mathrm{St}_{n d}$. Using this, one can compare the singularities of, for instance, $(z d / d z)^{n}-z$ and $(u d / d u)^{n}-n^{n} u^{n}$ where $z=u^{n}$.

Monodromy identity. Let the differential module $M$ over $\mathbb{C}\left(\left\{z^{-1}\right\}\right)$ correspond to the tuple $\left(V,\left\{V_{q}\right\}, \gamma,\left\{\mathrm{St}_{d}\right\}\right)$. Let $W$ be a solution space at a certain point $p$ close to $z=\infty$. One makes a loop around $z=\infty$ and analytic continuation along this loop yields the topological monodromy $\operatorname{mon}_{\infty} \in \mathrm{GL}(W)$. After some identification of $W$ with $V$ one obtains the monodromy identity, see [10, Proposition 8.12]

$$
\text { mon }_{\infty} \text { is conjugated to } \gamma \prod_{\substack{d \in[0,1) \\ d \text { singular }}} \mathrm{St}_{d}
$$

where the order of the maps $\mathrm{St}_{d}$ in the product is counter clockwise.

\section{Stokes matrices for $\delta^{n}-z$}

We summarize the results for the quantum differential operator $\delta^{n}-z$ which is the quantum differential operator of $\mathbb{P}^{n-1}$ (normalized by putting $\hbar=1$ ). The irregular singular point $z=\infty$ has (generalized) eigenvalues $q_{j}=e^{2 \pi i j / n} z^{1 / n}, j=0, \ldots, n-1$.

The symbolic solution space $V$ at $z=\infty$ has a basis $e_{0}, \ldots, e_{n-1}$, uniquely determined (up to simultaneous multiplication by a constant) by normalizing the matrix of $\gamma$. 
Let $E_{k \ell} \in \operatorname{End}(V)$ denote the map defined by $E_{k \ell} e_{\ell}=e_{k}$ and $E_{k \ell} e_{j}=0$ for $j \neq \ell$. For a direction $d$, the Stokes matrix $\mathrm{St}_{d} \in \mathrm{GL}(V)$ has the form $\mathrm{St}_{d}=1+\sum x_{\ell k} E_{\ell k}$, where the sum is taken over the pairs $(k, \ell)$ such that the direction $d$ is singular for $q_{k}-q_{\ell}$. For $k \neq \ell$ the pair $\left(q_{k}, q_{\ell}\right)$ has in the interval $[0, n)$ precisely one singular direction and produces the constant $x_{\ell k}$. Among $n(n-1)$ singular directions in $[0, n)$ (counted with multipicity) there are $n-1$ in the interval $[0,1)$. The values $x_{\ell k}$ corresponding to the singular directions in $[0,1)$ are computed using the monodromy identity. The other $x_{\ell k}$ are obtained by the formula $\gamma^{-1} \mathrm{St}_{d} \gamma=\mathrm{St}_{d+1}$.

The 'Stokes data' for the equation is by definition $\left\{x_{\ell k}\right\}_{k \neq \ell}$. We note that $x_{\ell k}=x_{\ell^{\prime} k^{\prime}}$ if $\ell \equiv \ell^{\prime}, k \equiv k^{\prime} \bmod n$. The result of this section is

Theorem 3.1 The monodromy identity yields the following formulas:

For $n$ odd,

$$
x_{l k}=\left\{\begin{array}{l}
-(-1)^{k-l}\left(\begin{array}{c}
n \\
k-l
\end{array}\right) \quad \text { for } n>k>l \geq 0, k+l=\left[\begin{array}{l}
n \\
2
\end{array}\right] \text { or }\left[\frac{n}{2}\right]-1, \\
(-1)^{l-k}\left(\begin{array}{c}
n \\
l-k
\end{array}\right) \quad \text { for } n>l>k \geq 0, k+l=3\left[\begin{array}{c}
\frac{n}{2} \\
l
\end{array}\right]+1 \text { or } 3\left[\begin{array}{c}
\frac{n}{2} \\
\end{array}\right],
\end{array}\right.
$$

and $x_{l+s, k+s}=x_{l k}$ for all $s \in \mathbb{Z}$.

For $n$ even,

$$
x_{l k}= \begin{cases}-(-1)^{k-l}\left(\begin{array}{c}
n \\
k-l
\end{array}\right) & \text { for } n>k>l \geq 0, \quad k+l=\frac{n}{2} \text { or } \frac{n}{2}-1, \\
(-1)^{l-k}\left(\begin{array}{c}
n \\
l-k
\end{array}\right) & \text { for } n>l>k \geq 0, \quad k+l=3 \frac{n}{2} \text { or } 3 \frac{n}{2}-1,\end{cases}
$$

and $x_{l+s, k+s}=x_{l k}$ for all $s \in \mathbb{Z}$.

From the above one deduces for $0 \leq k, \ell<n, k \neq \ell$, the formulas:

- For $n$ odd and $0 \leq \ell<k$ one has $x_{\ell k}=-(-1)^{k-\ell}\left(\begin{array}{c}n \\ k-\ell\end{array}\right)$ and $x_{\ell k}=-x_{k \ell}$.

- For $n$ even and $0 \leq \ell<k$ one has $x_{\ell k}=-(-1)^{k-\ell}\left(\begin{array}{c}n \\ k-\ell\end{array}\right)$ if $k-\ell \leq n / 2$ and $x_{\ell k}=(-1)^{k-\ell}\left(\begin{array}{c}n \\ k-\ell\end{array}\right)$ if $k-\ell>n / 2$.

- For $n$ even and $0 \leq k<\ell$ one has $x_{\ell k}=(-1)^{\ell-k}\left(\begin{array}{c}n \\ \ell-k\end{array}\right)$.

The second part of Theorem 3.1 is obtained from the first part by using the equalities $x_{\ell k}=x_{\ell^{\prime} k^{\prime}}$ if $\ell \equiv \ell^{\prime}, k \equiv k^{\prime} \bmod n$ and the equalities $x_{\ell k}=x_{\ell+s, k+s}$ for all $s \in \mathbb{Z}$.

\subsection{Generalised eigenvalues and formal monodromy}

The scalar operator $(z d / d z)^{n}-z$ can be transformed into a matrix differential operator $(z d / d z)+A$, where the entries of the matrix $A$ are in $\mathbb{C}\left(\left\{z^{-1}\right\}\right)$. More precisely, the matrix $A$ has the form 


$$
\left(\begin{array}{ccccccc}
0 & 1 & 0 & \ldots & 0 & 0 & 0 \\
0 & 0 & 1 & \ldots & 0 & 0 & 0 \\
\vdots & \vdots & \ddots & \ddots & \vdots & \vdots & \vdots \\
\vdots & \vdots & \vdots & \ddots & \ddots & \vdots & \vdots \\
0 & 0 & 0 & \ldots & 0 & 1 & 0 \\
0 & 0 & 0 & \ldots & 0 & 0 & 1 \\
z & 0 & 0 & \ldots & 0 & 0 & 0
\end{array}\right) .
$$

In the case of $(z d / d z)^{n}-z$, the differential module $\mathbb{C}\left(\left(z^{-1 / n}\right)\right) \otimes M$ has a basis $b_{0}, \ldots, b_{n-1}$ such that $\delta b_{j}=-q_{j} b_{j}$ with $q_{j}=\zeta^{j} z^{1 / n}$ and $\zeta=e^{2 \pi i / n}$. The values $q_{j}$ are the generalized eigenvalues and the matrix form of $\delta$, with respect to this basis, reads $z d / d z-\operatorname{diag}\left(z^{1 / n}, \zeta z^{1 / n}, \ldots, \zeta^{n-1} z^{1 / n}\right)$.

The symbolic solution space $V$ has the basis

$$
\left\{e_{j}=e^{\zeta^{j} z^{1 / n}} \frac{b_{j}}{n}: j=0, \ldots, n-1\right\} .
$$

The elements $b_{j}$ are unique up to multiplication by a constant. From the identities $\gamma V_{q}=V_{\gamma q}$ it follows that these constants are chosen so that the formal monodromy $\gamma$ has the form $e_{0} \mapsto e_{1} \mapsto \cdots \mapsto e_{n-2} \mapsto e_{n-1} \mapsto(-1)^{n} e_{0}$. The sign $(-1)^{n}$ comes from the observation that $\gamma$ has determinant 1 on $V$.

In this case $\operatorname{mon}_{\infty}$ can be identified with the topological monodromy mon 0 at $z=0$ (because $\mathbb{Z}$ is the fundamental group of $\mathbb{C}^{*}$ ). This is a unipotent matrix with characteristic polynomial $(\lambda-1)^{n}$.

\subsection{Singular directions}

Put $\left(\zeta^{k}-\zeta^{\ell}\right)=\left|\zeta^{k}-\zeta^{\ell}\right| \cdot e^{2 \pi i \phi(k, \ell)}$ with, say, $0 \leq \phi(k, \ell)<1$. Now, $d$ is a singular direction for $q_{k}-q_{\ell}$ if and only if $\cos (2 \pi \phi(k, \ell)+2 \pi d / n)=-1$. Thus $d=d(k, l)=n(1 / 2-\phi(k, \ell))$ is modulo $n$ the only singular direction for the pair $\left(q_{k}, q_{l}\right)$.

Recall that the symbolic solution space $V$ has basis $e_{0}, \ldots, e_{n-1}$. We denote by $E_{a b} \in \operatorname{End}(V)$ the map given by $E_{a b} e_{b}=e_{a}$ and $E_{a b} e_{c}=0$ for $c \neq b$. One has $E_{a b} E_{b c}=E_{a c}$. Moreover, the part of $\operatorname{St}_{d(k, l)}$ corresponding to the pair $\left(q_{k}, q_{l}\right)$ has the form $x_{l k} E_{l k}$ for a certain constant $x_{l k}$. Then

$$
\mathrm{St}_{d}=1+\sum_{\substack{(k, l) \\ d=d(k, l) \bmod n}} x_{l k} E_{l k} .
$$

Our goal is to compute all constants $x_{l k}$.

Computation of $d(k, l)$. One observes that for $\lambda \in(0,1) \subset \mathbb{R}$, the formula $\left(e^{2 \pi i \lambda}-\right.$ $1)=\left|\left(e^{2 \pi i \lambda}-1\right)\right| e^{2 \pi i \mu}$ holds with $\mu=1 / 4+\lambda / 2$. This implies: 
- For $n>k>l \geq 0$ one has $\phi(k, l)=1 / 4+(k+l) / 2 n$ and $d(k, l)=n / 4-(k+l) / 2$. For $n>l>k \geq 0$ one has $\phi(k, l)=3 / 4+(k+l) / 2 n$ and $d(k, l)=3 n / 4-(k+$ l) $/ 2$.

- For $n$ odd and $n>k>l \geq 0$, the possibilities for $d(k, l) \in[0,1)+\mathbb{Z} n$ are given by: $k+l=[n / 2], d(k, l)=1 / 4$ and $k+l=[n / 2]-1, d(k, l)=3 / 4$.

- For $n$ odd and $n>l>k \geq 0$, the possibilities for $d(k, l) \in[0,1)+\mathbb{Z} n$ are given by: $k+l=3[n / 2]+1, d(k, l)=1 / 4$ and $k+l=3[n / 2], d(k, l)=3 / 4$.

- For $n$ even and $n>k>l \geq 0$, the possibilities for $d(k, l) \in[0,1)+\mathbb{Z} n$ are given by: $k+l=n / 2, d(k, l)=0$ and $k+l=n / 2-1, d(k, l)=1 / 2$.

- For $n$ even and $n>l>k \geq 0$, the possibilities for $d(k, l) \in[0,1)+\mathbb{Z} n$ are given by: $k+l=3 n / 2, d(k, l)=0$ and $k+l=3 n / 2-1, d(k, l)=1 / 2$.

\subsection{Equation for odd $n$}

The monodromy identity for odd $n$ is: $\operatorname{mon}_{\infty}$ is conjugated to $\gamma \mathrm{St}_{3 / 4} \mathrm{St}_{1 / 4}$. Therefore, $P_{n}=\operatorname{det}\left(-\lambda 1+\gamma \mathrm{St}_{3 / 4} \mathrm{St}_{1 / 4}\right)$ equals $-(\lambda-1)^{n}$. Further,

$$
\begin{aligned}
\gamma & =E_{10}+E_{21}+\cdots+E_{n-1, n-2}+E_{0, n-1}, \\
\mathrm{St}_{3 / 4} & =1+\sum_{\substack{k+l=[n / 2]-1 \\
k>l}} x_{l k} E_{l k}+\sum_{\substack{k+l=3[n / 2] \\
l>k}} x_{l k} E_{l k}, \\
\mathrm{St}_{1 / 4} & =1+\sum_{\substack{k+l=[n / 2] \\
k>l}} x_{l k} E_{l k}+\sum_{\substack{k+l=3[n / 2]+1 \\
l>k}} x_{l k} E_{l k} .
\end{aligned}
$$

One observes that $P_{n}$ is the determinant of a sparse matrix and guided by a few explicit examples, verified by a MAPLE computation,

$$
\begin{gathered}
P_{3}=-\lambda^{3}+x_{01} \lambda^{2}+x_{21} \lambda+1, \\
P_{5}=-\lambda^{5}+x_{01} \lambda^{4}+x_{02} \lambda^{3}+x_{42} \lambda^{2}+x_{43} \lambda+1, \\
P_{7}=-\lambda^{7}+x_{12} \lambda^{6}+x_{02} \lambda^{5}+x_{03} \lambda^{4}+x_{63} \lambda^{3}+x_{64} \lambda^{2}+x_{54} \lambda+1, \\
P_{9}=-\lambda^{9}+x_{12} \lambda^{8}+x_{13} \lambda^{7}+x_{03} \lambda^{6}+x_{04} \lambda^{5} \\
\quad+x_{84} \lambda^{4}+x_{85} \lambda^{3}+x_{75} \lambda^{2}+x_{76} \lambda+1 .
\end{gathered}
$$

one obtains the general formula for $P_{n}$ and odd $n$ :

$$
P_{n}=-\lambda^{n}+1+\sum_{\substack{k>l \\ k+l=[n / 2] \text { or }[n / 2]-1}} x_{l k} \lambda^{n-(k-l)}+\sum_{\substack{l>k \\ k+l=3[n / 2]+1 \text { or } 3[n / 2]}} x_{l k} \lambda^{l-k}
$$


From this and the equality $\gamma^{-1} \mathrm{St}_{d} \gamma=\mathrm{St}_{d+1}$ one obtains

$$
x_{l k}= \begin{cases}-(-1)^{k-l}\left(\begin{array}{c}
n \\
k-l
\end{array}\right) & \text { for } k>l, \quad k+l=\left[\frac{n}{2}\right] \text { or }\left[\frac{n}{2}\right]-1, \\
(-1)^{l-k}\left(\begin{array}{c}
n \\
l-k
\end{array}\right) & \text { for } l>k, \quad k+l=3\left[\frac{n}{2}\right]+1 \text { or } 3\left[\frac{n}{2}\right],\end{cases}
$$

and $x_{l+t, k+t}=x_{l k}$ for all $t \in \mathbb{Z}$.

The proof of the formula for $P_{n}$ consists simply of determining for each power of $\lambda$ the part of the sparse matrix which contributes to its coefficient in the determinant. The verification is straightforward.

\subsection{Equation for even $n$}

According to the monodromy identity, $\operatorname{mon}_{\infty}$ is conjugated to $\gamma \mathrm{St}_{1 / 2} \mathrm{St}_{0}$. Thus $P_{n}=$ $\operatorname{det}\left(-\lambda 1+\gamma \mathrm{St}_{1 / 2} \mathrm{St}_{0}\right)$ equals $(\lambda-1)^{n}$.

$$
\begin{aligned}
\gamma & =E_{10}+E_{21}+\cdots+E_{n-1, n-2}-E_{0, n-1}, \\
\mathrm{St}_{1 / 2} & =1+\sum_{\substack{k>l \\
k+l=n / 2-1}} x_{l k} E_{l k}+\sum_{\substack{l>k \\
k+l=3 n / 2-1}} x_{l k} E_{l k}, \\
\mathrm{St}_{0} & =1+\sum_{\substack{k>l \\
k+l=n / 2}} x_{l k} E_{l k}+\sum_{\substack{l>k \\
k+l=3 n / 2}} x_{l k} E_{l k} .
\end{aligned}
$$

Guided by a few examples, verified by a MAPLE computation,

$$
\begin{aligned}
& P_{2}=\lambda^{2}-x_{01} \lambda+1, \\
& P_{4}=\lambda^{4}-x_{01} \lambda^{3}-x_{02} \lambda^{2}+x_{32} \lambda+1, \\
& P_{6}=\lambda^{6}-x_{12} \lambda^{5}-x_{02} \lambda^{4}-x_{03} \lambda^{3}+x_{53} \lambda^{2}+x_{54} \lambda+1, \\
& P_{8}=\lambda^{8}-x_{12} \lambda^{7}-x_{13} \lambda^{6}-x_{03} \lambda^{5}-x_{04} \lambda^{4}+x_{74} \lambda^{3}+x_{75} \lambda^{2}+x_{65} \lambda+1,
\end{aligned}
$$

one deduces the general formula for $P_{n}$ and even $n$.

$$
P_{n}=\lambda^{n}+1-\sum_{\substack{k>l \\ k+l=n / 2 \text { or } n / 2-1}} x_{l k} \lambda^{n-(k-l)}+\sum_{\substack{l>k \\ k+l=3 n / 2 \text { or }=3 n / 2-1}} x_{l k} \lambda^{l-k} .
$$

This implies

$$
x_{l k}= \begin{cases}-(-1)^{k-l}\left(\begin{array}{c}
n \\
k-l
\end{array}\right) & \text { for } k>l, \quad k+l=\frac{n}{2} \text { or } \frac{n}{2}-1, \\
(-1)^{l-k}\left(\begin{array}{c}
n \\
l-k
\end{array}\right) & \text { for } l>k, \quad k+l=3 \frac{n}{2} \text { or } 3 \frac{n}{2}-1,\end{cases}
$$


and $x_{l+s, k+s}=x_{l k}$ for all $s \in \mathbb{Z}$.

Remark 3.2 (Weighted projective spaces) Consider positive integers $w_{0}, \ldots, w_{n}$ with $\operatorname{gcd}\left(w_{0}, \ldots, w_{n}\right)=1$. For the weighted projective space $\mathbb{P}\left(w_{0}, \ldots, w_{n}\right)$, which is defined by $\mathbb{C}^{n+1} \backslash\{0\} / \mathbb{C}^{*}$, where $t \cdot\left(z_{0}, \ldots, z_{n}\right)=\left(t^{w_{0}} z_{0}, \ldots, t^{w_{n}} z_{n}\right)$, we adopt the quantum differential operator, given in [7], namely,

$$
\prod_{i=1}^{n}\left(w_{i} \hbar \partial\right)\left(w_{i} \hbar \partial-\hbar\right) \cdots\left(w_{i} \hbar-\left(w_{i}-1\right) \hbar\right)-q,
$$

where $\partial=q d / d q$. After taking $\hbar=1$ and replacing $q$ by $z$ and $\partial$ by $\delta=z d / d z$ the operator reads

$$
\prod_{j=0}^{n} \delta\left(\delta-\frac{1}{w_{j}}\right) \cdots\left(\delta-\frac{w_{j}-1}{w_{j}}\right)-z .
$$

We note that the above formula is attributed to Corti and Golyshev and that in [9] Dubrovin's conjecture is extended to orbifolds. In particular, there is a conjecture for weighted projective spaces. Unfortunately, the latter is not explicit enough to allow us a comparison with the Stokes data. Here we will show that our computations of the classical Stokes matrices for ordinary projective spaces extend to the case of weighted projective spaces. The preprint [12], related to Proposition 3.3, appeared after this paper was finished.

Proposition 3.3 Let $\left\{x_{\ell k}\right\}$ be the Stokes data for (1). Put $s=\sum w_{j}$. At $z=\infty$, the generalized eigenvalues are $\zeta^{j} z^{1 / s}$ with $j=0, \ldots, s-1$, where $\zeta=e^{2 \pi i / s}$. Thus the above operator is formally equivalent to $\delta^{s}-z$ and the configuration of the Stokes matrices is the same as for the ordinary projective space $\mathbb{P}^{s-1}$. The formal monodromy differs by a minus-sign if $n$ is even.

The topological monodromy at $z=0$ (or equivalently at $z=\infty$ ) has characteristic polynomial $\prod_{j=0}^{n}\left(\lambda^{w_{j}}-1\right)$. The Stokes data $\left\{x_{\ell k}\right\}$ are determined by

- The monodromy identity $\pm P_{n}=\prod_{j=0}^{n}\left(\lambda^{w_{j}}-1\right)$.

- $x_{\ell k}=x_{\ell^{\prime} k^{\prime}}$ if $\ell \equiv \ell^{\prime}, k \equiv k^{\prime} \bmod s$.

- $x_{\ell+t, k+t}=x_{\ell k}$ for all $t \in \mathbb{Z}$.

In particular, the Stokes data consists of computable integers.

The proof is a straightforward computation. We note that it might be difficult to give a closed formula (as in the $\mathbb{P}^{n-1}$ case) for the $x_{\ell k}$.

Example $3.4(\mathbb{P}(1,2,4))$ The topological monodromy at $z=\infty$ is conjugated to $\gamma \mathrm{St}_{3 / 4} \mathrm{St}_{1 / 4}$. The characteristic polynomial of this $7 \times 7$-matrix is $-\lambda^{7}+x_{12} \lambda^{6}+$ $x_{02} \lambda^{5}+x_{03} \lambda^{4}+x_{63} \lambda^{3}+x_{64} \lambda^{2}+x_{54} \lambda+1$, where these $x_{\ell k}$ are the nontrivial entries of $\mathrm{St}_{3 / 4}$ and $\mathrm{St}_{1 / 4}$.

The topological monodromy at $z=0$ has characteristic polynomial $-(\lambda-1)\left(\lambda^{2}-\right.$ 1) $\left(\lambda^{4}-1\right)$ and thus we find

$$
x_{12}=1, \quad x_{02}=1, \quad x_{03}=-1, \quad x_{63}=1, \quad x_{64}=-1, \quad x_{54}=-1 .
$$


4 Quantum differential operator $\delta^{N-1}-z k(k \delta+k-1)(k \delta+k-2) \cdots(k \delta+1)$

According to [5], the Dubrovin-Givental connection for a nonsingular hypersurface of degree $k \leq N-1$ in $\mathbb{P}^{N-1}$ is given by $\delta^{N-1}-z k(k \delta+k-1)(k \delta+k-2) \cdots(k \delta+1)$. We prefer to write this operator differently (with $m=k$ and $n=N-k$ ) as

$$
\delta^{n+m-1}-m^{m} z\left(\delta+\frac{m-1}{m}\right)\left(\delta+\frac{m-2}{m}\right) \cdots\left(\delta+\frac{1}{m}\right),
$$

$\delta=z d / d z, n>1, m>1$. For $m=1$ we get the operator studied in Sect. 3. At the end of this section we will comment on the case $n=1$.

Theorem 4.1 The Stokes data for (2) is $\left\{x_{\ell k}: 0 \leq k, \ell \leq n-1, k \neq \ell\right\}$ and $\left\{z_{j}: 1 \leq j \leq m-1\right\},\left\{y_{j}: 1 \leq j \leq m-1\right\}$. The values $y_{j}$ and $z_{j}$ depend on the choice of a basis. However, the products $y_{j} z_{j}, j=1, \ldots, m-1$, are computable elements of $\mathbb{Q}(\zeta)$, where $\zeta=e^{2 \pi i / m}$, and independent of this choice. Equality $x_{\ell+s, k+s}=x_{\ell k}$ holds for $s \in \mathbb{Z}$ and $x_{\ell k}=x_{\ell^{\prime} k^{\prime}}$ if $\ell \equiv \ell^{\prime}, k \equiv k^{\prime} \bmod n$.

For $n>1$ odd,

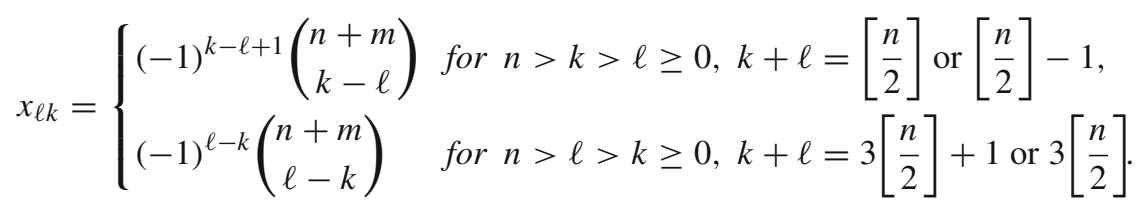

For $n$ even,

$$
x_{\ell k}=\left\{\begin{array}{l}
(-1)^{k-\ell+1}\left(\begin{array}{c}
n+m \\
k-\ell
\end{array}\right) \quad \text { for } n>k>\ell \geq 0, k+\ell=\frac{n}{2} \text { or } \frac{n}{2}-1, \\
(-1)^{n+m+\ell-k+1}\left(\begin{array}{c}
n+m \\
\ell-k
\end{array}\right) \text { for } n>\ell>k \geq 0, k+\ell=3 \frac{n}{2} \text { or } 3 \frac{n}{2}-1 .
\end{array}\right.
$$

4.1 Differential operator $\delta^{4}-27 z \delta^{2}-27 z \delta-6 z$

We start by investigating the case $n=2, m=3$ of Theorem 4.1, which is the quantum differential equation of a hypersurface of degree 3 in $\mathbb{P}^{4}$, see [5, p.42, Example 3.6]. A matrix form for this equation is

$$
z \frac{d}{d z}+\left(\begin{array}{cccc}
0 & -1 & 0 & 0 \\
0 & 0 & -1 & 0 \\
0 & 0 & 0 & -1 \\
-6 z & -27 z & -27 z & 0
\end{array}\right)
$$

We proceed as in Sect. 3. The (generalised) eigenvalues at $z=\infty$ are $q_{1}=\sqrt{27} z^{1 / 2}$, $q_{2}=-\sqrt{27} z^{1 / 2}, 0$. The symbolic solution space $V$ at $z=\infty$ has the form $V=$ 
$V_{q_{1}} \oplus V_{q_{2}} \oplus V_{0}$ with $V_{q_{1}}=\mathbb{C} e_{1}, V_{q_{2}}=\mathbb{C} e_{2}$ and $V_{0}=\mathbb{C} e_{3} \oplus \mathbb{C} e_{4}$. The basis $e_{1}, \ldots, e_{4}$ is chosen so that the formal monodromy has the form

$$
\gamma=\left(\begin{array}{cccc}
0 & -1 & 0 & 0 \\
1 & 0 & 0 & 0 \\
0 & 0 & \zeta & 0 \\
0 & 0 & 0 & \zeta^{2}
\end{array}\right), \quad \zeta=e^{2 \pi i / 3}
$$

We note that this basis is unique up to a transformation of the type $e_{1} \mapsto \lambda_{1} e_{1}$, $e_{2} \mapsto \lambda_{1} e_{2}, e_{3} \mapsto \lambda_{2} e_{3}, e_{4} \mapsto \lambda_{3} e_{4}$ with all $\lambda_{j} \in \mathbb{C}^{*}$.

The singular directions are $0+2 \mathbb{Z}$ for the differences $q_{2}-q_{1}, q_{2}-0,0-q_{1}$ and are $1+2 \mathbb{Z}$ for the differences $q_{1}-q_{2}, q_{1}-0,0-q_{2}$. The Stokes matrix $\mathrm{St}_{0}$ has the form

$$
\mathrm{St}_{0}=\left(\begin{array}{cccc}
1 & 0 & x_{4} & x_{5} \\
x_{1} & 1 & 0 & 0 \\
0 & x_{2} & 1 & 0 \\
0 & x_{3} & 0 & 1
\end{array}\right), \quad \gamma \mathrm{St}_{0}=\left(\begin{array}{cccc}
-x_{1} & -1 & 0 & 0 \\
1 & 0 & x_{4} & x_{5} \\
0 & \zeta x_{2} & \zeta & 0 \\
0 & \zeta^{2} x_{3} & 0 & \zeta^{2}
\end{array}\right)
$$

and $\mathrm{St}_{1}=\gamma^{-1} \mathrm{St}_{0} \gamma$. According to the monodromy identity, $\gamma \mathrm{St}_{0}$ is equivalent to the topological monodromy at $z=0$. The latter is seen to have the single eigenvalue 1 (and only one Jordan block). Thus, the characteristic polynomial of $\gamma \mathrm{St}_{0}$ is $(\lambda-1)^{4}$. This yields the data for the entries of the Stokes matrices $x_{1}=-5, x_{2} x_{4}=-9 \zeta+18$, $x_{3} x_{5}=9 \zeta+27$. It seems that we have not enough information to obtain values for all $x_{j}$. This is due, however, to the nonuniqueness of the basis vectors $e_{3}, e_{4}$. As an example we can see that for a suitable choice of $e_{3}, e_{4}$ we will have, say, $x_{4}=1$ and $x_{5}=1$ and further $x_{1}=-5, x_{2}=-9 \zeta+18, x_{3}=9 \zeta+27$.

\subsection{General case}

The above operator is transformed in the usual way into a first order matrix differential operator. The formal data for the symbolic solution space $V$ at $z=$ $\infty$ are: the (generalised) eigenvalues are 0 and $q_{j}=\sqrt[n]{m^{m}} \zeta_{n}^{j} z^{1 / n}$ for $j=$ $0,1, \ldots, n-1$ with $\zeta_{n}=e^{2 \pi i / n}$. This solution space $V$ has the decomposition $V=V_{q_{0}} \oplus V_{q_{1}} \oplus \cdots \oplus V_{q_{n-1}} \oplus V_{0}$ with $V_{q_{j}}=\mathbb{C} e_{j}$ for $j=0, \ldots, n-1$ and $V_{0}=\mathbb{C} f_{1} \oplus \cdots \oplus \mathbb{C} f_{m-1}$. The basis vectors are chosen so that the formal monodromy $\gamma$ acts as $e_{0} \mapsto e_{1} \mapsto \cdots \mapsto e_{n-1} \mapsto(-1)^{n-1}(-1)^{m-1} e_{0}$ and $\gamma f_{j}=\zeta_{m}^{j} f_{j}$ for $j=1, \ldots, m-1$ and $\zeta_{m}=e^{2 \pi i / m}$. We note that the basis $f_{1}, \ldots, f_{m-1}$ of $V_{0}$ is unique up to multiplication by scalars. The computation of the 'monodromy identity' is done separately for $n$ even and $n$ odd.

\section{Even $n$.}

The singular directions $d$ for $q_{k}-q_{\ell}$ lying in $[0,1)+\mathbb{Z} n$ are the same as in Sect. 3 , namely:

- For $n>k>\ell \geq 0: d=0$ and $k+\ell=n / 2 ; d=1 / 2$ and $k+\ell=n / 2-1$. 
- For $n>\ell>k \geq 0: d=0$ and $k+\ell=3 n / 2 ; d=1 / 2$ and $k+\ell=3 n / 2-1$.

- For $q_{k}-0$, the only singular direction in $[0,1)+\mathbb{Z} n$ is $d=0$ with $k=n / 2$.

- For $0-q_{k}$, the only singular direction in $[0,1)+\mathbb{Z} n$ is $d=0$ with $k=0$.

Description of $\mathrm{St}_{0}$. For elements in $\operatorname{End}\left(\mathbb{C} e_{0}+\cdots+\mathbb{C} e_{n-1}\right)$ we use the notation of Sect. 3. Then $\mathrm{St}_{0}$ is the identity plus a number of maps, namely, $\sum_{k>\ell, k+\ell=n / 2} x_{\ell k} E_{\ell, k}$ and $\sum_{\ell>k, k+\ell=3 n / 2} x_{\ell k} E_{\ell k}$ and a map $e_{n / 2} \mapsto y_{1} f_{1}+\cdots+y_{m-1} f_{m-1}$ (the other base vectors are mapped to 0 ) and for $j=1, \ldots, m-1$ a map $f_{j} \mapsto z_{j} e_{0}$ (the other base vectors are mapped to 0 ).

Description of $\mathrm{St}_{1 / 2}$. This Stokes matrix is the identity plus certain maps, namely, $\sum_{k>\ell, k+\ell=n / 2-1} x_{\ell k} E_{\ell k}$ and $\sum_{\ell>k, k+\ell=3 n / 2-1} x_{\ell k} E_{\ell k}$.

The matrix $\gamma \mathrm{St}_{1 / 2} \mathrm{St}_{0}$ and its characteristic polynomial $P$ can be computed. The monodromy identity $P=(\lambda-1)^{n+m-1}$ leads to the statement that $x_{\ell k}$ have the form $\pm\left(\begin{array}{c}n+m \\ *\end{array}\right)$ and that the $y_{j} z_{j}$ are elements of $\mathbb{Q}\left[\zeta_{m}\right]$. As in Sect. 4.1, i.e., the case $n=2$, $m=3$, one cannot compute $y_{j}$ and $z_{j}$ separately since this involves a definite choice of the basis $f_{1}, \ldots, f_{m-1}$.

Example 4.2 The case $n=4, m=3$ and $\zeta=e^{2 \pi i / 3}$.

$$
\gamma=\left(\begin{array}{cccccc}
0 & 0 & 0 & -1 & 0 & 0 \\
1 & 0 & 0 & 0 & 0 & 0 \\
0 & 1 & 0 & 0 & 0 & 0 \\
0 & 0 & 1 & 0 & 0 & 0 \\
0 & 0 & 0 & 0 & \zeta & 0 \\
0 & 0 & 0 & 0 & 0 & \zeta^{2}
\end{array}\right), \quad \mathrm{St}_{1 / 2}=\left(\begin{array}{cccccc}
1 & x_{01} & 0 & 0 & 0 & 0 \\
0 & 1 & 0 & 0 & 0 & 0 \\
0 & 0 & 1 & 0 & 0 & 0 \\
0 & 0 & x_{32} & 1 & 0 & 0 \\
0 & 0 & 0 & 0 & 1 & 0 \\
0 & 0 & 0 & 0 & 0 & 1
\end{array}\right),
$$

$$
\mathrm{St}_{0}=\left(\begin{array}{cccccc}
1 & 0 & x_{02} & 0 & z_{1} & z_{2} \\
0 & 1 & 0 & 0 & 0 & 0 \\
0 & 0 & 1 & 0 & 0 & 0 \\
0 & 0 & 0 & 1 & 0 & 0 \\
0 & 0 & y_{1} & 0 & 1 & 0 \\
0 & 0 & y_{2} & 0 & 0 & 1
\end{array}\right)
$$

Since the characteristic polynomial of $\gamma \mathrm{St}_{1 / 2} \mathrm{St}_{0}$ is $(\lambda-1)^{6}$, one finds $x_{01}=7$, $x_{02}=-21, x_{32}=-7, y_{1} z_{1}=9\left(2 \zeta^{2}+1\right), y_{2} z_{2}=-9\left(2 \zeta^{2}+1\right)$.

Let $P$ denote again the characteristic polynomial of $\gamma \mathrm{St}_{1 / 2} \mathrm{St}_{0}$ for $n$ even and $m>1$. One observes that $(\lambda-1)^{n+m}=(\lambda-1) P$ is the sum of $\left(\lambda^{m}-1\right) Q$ with

$$
Q=\lambda^{n}-\sum_{\substack{k>l \\ k+l=n / 2 \text { or } n / 2-1}} x_{l k} \lambda^{n-(k-l)}+\sum_{\substack{l>k \\ k+l=3 n / 2 \text { or } 3 n / 2-1}} x_{l k} \lambda^{l-k}+1
$$


and terms $a \lambda^{j}, a \in \mathbb{C}$, with $3 n / 2<j<m+n / 2$. This leads to the formulas

$$
x_{\ell k}= \begin{cases}(-1)^{k-\ell+1}\left(\begin{array}{l}
n+m \\
k-\ell
\end{array}\right) & \text { for } k>\ell, \quad k+\ell=\frac{n}{2} \text { or } \frac{n}{2}-1, \\
(-1)^{n+m+\ell-k+1}\left(\begin{array}{c}
n+m \\
\ell-k
\end{array}\right) & \text { for } \ell>k, \quad k+\ell=3 \frac{n}{2} \text { or } 3 \frac{n}{2}-1 .\end{cases}
$$

The elements $y_{j} z_{j}$ are (in general complicated) expressions in $\mathbb{Q}(\zeta)$.

$\operatorname{Odd} n>1$.

The singular directions $d$ in $[0,1)+\mathbb{Z n}$ are

- For $q_{k}-q_{\ell}$ :

- $n>k>\ell \geq 0, d=1 / 4$ with $k+\ell=[n / 2] ; d=3 / 4$ with $k+\ell=[n / 2]-1$,

$-n>\ell>k \geq 0, d=1 / 4$ with $k+\ell=3[n / 2]+1 ; d=3 / 4$ with $k+\ell=3[n / 2]$.

- For $q_{k}-0: d=1 / 2$ and $k=[n / 2]$.

- For $0-q_{k}: d=0$ and $k=0$.

Example 4.3 The case $n=3, m=3, \zeta=e^{2 \pi i / 3}$.

$$
\begin{gathered}
\gamma=\left(\begin{array}{ccccc}
0 & 0 & 1 & 0 & 0 \\
1 & 0 & 0 & 0 & 0 \\
0 & 1 & 0 & 0 & 0 \\
0 & 0 & 0 & \zeta & 0 \\
0 & 0 & 0 & 0 & \zeta^{2}
\end{array}\right), \quad \mathrm{St}_{3 / 4}=\left(\begin{array}{cccccc}
1 & 0 & 0 & 0 & 0 \\
0 & 1 & 0 & 0 & 0 \\
0 & x_{21} & 1 & 0 & 0 \\
0 & 0 & 0 & 1 & 0 \\
0 & 0 & 0 & 0 & 1
\end{array}\right), \quad\left(\begin{array}{ccccc}
1 & x_{01} & 0 & 0 & 0 \\
0 & 1 & 0 & 0 & 0 \\
0 & 0 & 1 & 0 & 0 \\
0 & 0 & 0 & 1 & 0 \\
0 & 0 & 0 & 0 & 1
\end{array}\right), \quad \mathrm{St}_{0}=\left(\begin{array}{ccccccc}
1 & 0 & 0 & 0 & 0 \\
0 & 1 & 0 & 0 & 0 \\
0 & 0 & 1 & 0 & 0 \\
0 & z_{1} & 0 & 1 & 0 \\
0 & z_{2} & 0 & 0 & 1
\end{array}\right), \quad \mathrm{St}_{1 / 4}=\left(\begin{array}{ccccc}
1 & 0 & y_{1} & y_{2} \\
0 & 0 & 1 & 0 & 0 \\
0 & 0 & 0 & 1 & 0 \\
0 & 0 & 0 & 0 & 1
\end{array}\right) .
\end{gathered}
$$

The observation that the characteristic polynomial of $\gamma \mathrm{St}_{3 / 4} \mathrm{St}_{1 / 2} \mathrm{St}_{1 / 4} \mathrm{St}_{0}$ is $(\lambda-1)^{5}$ yields $x_{01}=6, x_{21}=-6$ and $y_{1} z_{1}=-9\left(\zeta^{2}+1\right), y_{2} z_{2}=9 \zeta^{2}$.

As in the case of even $n$ one obtains for general odd $n>1$ and $m>1$ explicit formulas for the entries $x_{\ell k}$ (the same notation as in the even case) of the Stokes matrices, namely,

$$
x_{\ell k}=\left\{\begin{array}{lll}
(-1)^{k-\ell+1}\left(\begin{array}{c}
n+m \\
k-\ell
\end{array}\right) & \text { for } k>\ell, & k+\ell=\left[\frac{n}{2}\right] \text { or }\left[\frac{n}{2}\right]-1, \\
(-1)^{\ell-k}\left(\begin{array}{c}
n+m \\
\ell-k
\end{array}\right) & \text { for } \ell>k, & k+\ell=3\left[\frac{n}{2}\right]+1 \text { or } 3\left[\frac{n}{2}\right] .
\end{array}\right.
$$

The elements $y_{j} z_{j}$ are (in general complicated) expressions in $\mathbb{Q}(\zeta)$.

Comments on the case $n=1$. In this case the operator is

$$
\delta^{m}-m^{m} z\left(\delta+\frac{m-1}{m}\right)\left(\delta+\frac{m-2}{m}\right) \cdots\left(\delta+\frac{1}{m}\right) .
$$


The (generalized) eigenvalues at $z=\infty$ are $q_{0}$ and 0 . This equation is not really a quantum differential equation and, moreover, there is no ramification at $z=\infty$.

For the symbolic solution space $V$ there is given a basis $e_{0}, f_{1}, \ldots, f_{m-1}$ such that $V_{q_{0}}=\mathbb{C} e_{0}, V_{0}$ has basis $f_{1}, \ldots, f_{m-1}$ and the formal monodromy $\gamma$ has the form $\gamma\left(e_{0}\right)=e_{0}$ and $\gamma\left(f_{j}\right)=\zeta^{j} f_{j}$ for all $j$ and $\zeta=e^{2 \pi i / m}$. The above basis is unique up to multiplication by scalars. The singular directions are $d=1 / 2$ and $d=0$ and the corresponding Stokes matrices involve (using the earlier notation) only $\left\{y_{1}, \ldots, y_{m-1}\right\}$ and $\left\{z_{1}, \ldots, z_{m-1}\right\}$. These elements are not unique, however, the products $y_{j} z_{j}$ are independent of the choice of $e_{0}, f_{1}, \ldots, f_{m-1}$ and are computable elements of $\mathbb{Q}(\zeta)$.

Example 4.4 For $m=3$ one finds $y_{1} z_{1}=3+3 \zeta, y_{2} z_{2}=-3 \zeta$. This example seems unrelated to the quantum cohomology of a cubic surface, studied by Ueda in [14].

Acknowledgments The first author thanks Hiroshi Iritani for pointing out the reference [11] and for many interesting conversations about the quantum cohomology of Fano varieties. We thank the referees for their helpful comments.

\section{References}

1. Cecotti, S., Vafa, C.: On classification of $N=2$ supersymmetric theories. Comm. Math. Phys. 158(3), 569-644 (1993)

2. Dubrovin, B.: Geometry of 2D topological field theories. In: Francaviglia, M., Greco, S. (eds.) Integrable Systems and Quantum Groups. Lecture Notes in Mathematics, vol. 1620, pp. 120-348. Springer, Berlin (1996)

3. Dubrovin, B.: Geometry and analytic theory of Frobenius manifolds. Doc. Math Extra II, 315-326 (1998)

4. Dubrovin, B.: Painlevé transcendents in two-dimensional topological field theory. In: Conte, R. (ed.) The Painlevé Property. CRM Series in Mathematical Physics, pp. 287-412. Springer, New York (1999)

5. Guest, M.A.: From Quantum Cohomology to Integrable Systems. Oxford Graduate Texts in Mathematics, vol. 15. Oxford University Press, Oxford (2008)

6. Guest, M.A., Its, A.R., Lin C.-S.: Isomonodromy aspects of the $\mathrm{tt}^{*}$ equations of Cecotti and Vafa I. Stokes data (2012). arXiv:1209.2045v1

7. Guest, M.A., Sakai, H.: Orbifold quantum D-modules associated to weighted projective spaces (2008). arXiv:0810.4236v1

8. Guzzetti, D.: Stokes matrices and monodromy of the quantum cohomology of projective spaces. Comm. Math. Phys. 207(2), 341-383 (1999)

9. Iritani, H.: An integral structure in quantum cohomology and mirror symmetry for toric orbifolds. Adv. Math. 222(3), 1016-1079 (2009)

10. van der Put, M., Singer, M.F.: Galois Theory of Linear Differential Equations. Grundlehren der Mathematischen Wissenschaften, vol. 328. Springer, Berlin (2003)

11. Tanabé, S.: Invariants of hypergeometric groups for Calabi-Yau complete intersections in weighted projective spaces. Bull. Sci. Math. 128(10), 811-827 (2004)

12. Tanabé, S., Ueda, K.: Invariants of hypergeometric groups for Calabi-Yau complete intersections in weighted projective spaces (2013). arXiv:1305.1659v2

13. Ueda, K.: Stokes matrices for the quantum cohomologies of Grassmannians. Int. Math. Res. Not. 34, 2075-2086 (2005)

14. Ueda, K.: Stokes matrix for the quantum cohomology of cubic surfaces (2005). arXiv:math/0505350v1

15. Zaslow, E.: Solitons and helices: the search for a math-physics bridge. Comm. Math. Phys. 175(2), 337-375 (1996) 